\title{
The left outflow tract in foetal echocardiography screening: an audit of practices and quality criteria.
}

\author{
Edwin Quarello ${ }^{1}$, Maud REGOUIN ${ }^{2}$, Julien MANCINI ${ }^{3}$, Antoine LAFOUGE ${ }^{4}$, Nathalie \\ FONTAINE $^{2}$, Stéphanie ROUSSIN ${ }^{1}$, Jimmy GUICHARD ${ }^{5}$, and Coralie DUMONT ${ }^{2}$
}

${ }^{1}$ Affiliation not available

${ }^{2}$ GHSR

${ }^{3} \mathrm{AMU}$

${ }^{4}$ Cabinet Hyères

${ }^{5}$ Cabinet d'échographie gynécologique et obstétricale

March 12, 2021

\begin{abstract}
Background: The detection rate of congenital heart defects is barely acceptable. Since 2016, the French National Conference on Obstetrical and Foetal Ultrasound updated its recommendations by the inclusion of an examination of the left ventricular outflow tract (LVOT) in the second and third trimester of pregnancy. Objectives: This study aimed on the one hand to evaluate the practices related to the realization of the LVOT in the setting of fetal echocardiography in low-risk populations, and on the other hand to study the possible modifications of the practices secondary to the introduction of quality criteria. Study Design: We conducted a multicentric, retrospective and prospective, descriptive, longitudinal study divided into three distinct periods: before 2016, in 2017, and in 2020. Seven quality criteria were investigated and rated from 0 to 1 for LVOT screening. Files were randomly selected from three centers, then average total and specific scores were calculated. Results: LVOT images were present in ultrasound reports in more than $93 \%$ of cases. Before 2016, the average quality score was 5.49/7 (95\% CI: 5.36-5.62), in $20175.91 / 7$ (95\% CI: 5.80-6.03), and in 2020 5.70/7 (95\% CI: 5.58-5.82) for the three centers. There was no significant difference following the introduction of the quality criteria; 2017 vs. 2020, p $=0.054$. Kappa coefficients of the inter- and intra-operator variables were all within 0.601 and 1 . Conclusion: Left ventricular outflow tract images were present in most of ultrasound reports. The introduction of the proposed quality criteria is not associated with a significant change in practice.
\end{abstract}

The left outflow tract in foetal echocardiography screening: an audit of practices and quality criteria.

Short Title: The left ventricular outflow tract in foetal echocardiography in low-risk populations.

Maud REGOUIN MD ${ }^{(1)}$, Julien MANCINI MD PhD ${ }^{(2)}$, Mr Antoine LAFOUGE ${ }^{(3)}$, Nathalie FONTAINE MD ${ }^{(1)}$, Stéphanie ROUSSIN MD ${ }^{(4)}$, Mr Jimmy GUICHARD ${ }^{(5)}$, Coralie DUMONT MD ${ }^{(1)}$, Edwin QUARELLO MD $\mathrm{PhD}^{(6,7)}$

(1): Département de Gynécologie Obstétrique, Hôpital Sud de la Réunion, Saint Pierre, Réunion, France.

(2): Aix Marseille Univ, APHM, INSERM, IRD, SESSTIM, Hop Timone, Public Health Department (BIOSTIC), 13005 Marseille, France Aix Marseille Univ, APHM, INSERM, IRD, SESSTIM, Hop Timone, Public Health Department (BIOSTIC), 13005 Marseille, France.

${ }^{(3)}$ : Cabinet de Gynécologie et Obstétrique, Hyères, France

(4): Clinique de l'Anjou, Pôle Mère-Enfant, 140 avenue de Lattre de Tassigny 49000 Angers 
${ }^{(5)}$ : Cabinet d'échographie gynécologique et obstétricale - Espace 9 mois - 15 boulevard Paul Vaillant Couturier 93100 MONTREUIL

(6) : Institut méditerranéen d'imagerie médicale appliquée à la gynécologie, la grossesse et l'enfance IMAGE², 6 Rue Rocca, 13008 Marseille, France.

(7) : Unité de dépistage et de diagnostic prénatal, hôpital Saint-Joseph, 26, boulevard de Louvain, 13285 Marseille cedex, France.

Correspondence:

Dr Edwin Quarello

Image2 Center

6 Rue Rocca

Marseille

France

e.quarello@me.com

. What are the novel findings of this work?

Introduction of quality criteria seems not always associated with improvement of practices

in the setting of fetal echocardiography in low-risk populations. Other strategies and tools must be developed.

. What are the clinical implications of this work?

In the setting of low-risk population, the left ventricular outflow tract is carried out in the majority of cases. Informativity of such view is not perfect and could be further improved.

\section{Abstract}

Background: The detection rate of congenital heart defects is barely acceptable. Since 2016, the French National Conference on Obstetrical and Foetal Ultrasound updated its recommendations by the inclusion of an examination of the left ventricular outflow tract (LVOT) in the second and third trimester of pregnancy.

Objectives: This study aimed on the one hand to evaluate the practices related to the realization of the LVOT in the setting of fetal echocardiography in low-risk populations, and on the other hand to study the possible modifications of the practices secondary to the introduction of quality criteria.

Study Design: We conducted a multicentric, retrospective and prospective, descriptive, longitudinal study divided into three distinct periods: before 2016, in 2017, and in 2020. Seven quality criteria were investigated and rated from 0 to 1 for LVOT screening. Files were randomly selected from three centers, then average total and specific scores were calculated.

Results: LVOT images were present in ultrasound reports in more than $93 \%$ of cases. Before 2016, the average quality score was 5.49/7 (95\% CI: 5.36-5.62), in 2017 5.91/7 (95\% CI: 5.80-6.03), and in 2020 5.70/7 (95\% CI: 5.58-5.82) for the three centers. There was no significant difference following the introduction of the quality criteria; 2017 vs. 2020, $\mathrm{p}=0.054$. Kappa coefficients of the inter- and intra-operator variables were all within 0.601 and 1 .

Conclusion: Left ventricular outflow tract images were present in most of ultrasound reports. The introduction of the proposed quality criteria is not associated with a significant change in practice.

Keywords:

Prenatal; fetal echocardiography; low-risk population; quality criteria; Artificial Intelligence

Introduction 
Prenatal screening for congenital heart defects (CHD) reduces the mortality and morbidity of newborns (1)(2), but also helps to improve their neuro-developmental outcome (3)(4). However, there is room for improvement in this screening which varies considerably, not only between countries, but also between regions within countries (5)(6)(7).

According to Sklansky et al. , the main reason for this shortcoming is that effective foetal echocardiography requires not only different images specific to the individual, as recommended by the national (CNEOF) and international (ISUOG, AUIM) learned societies, but also, and above all, good quality, informative images (8). The quality of cardiac screening examinations is the cornerstone to improve the detection rate of CHD in a low-risk population (9).

For more than 20 years, it has been recognized that screening for CHD can be improved by adding outflow tracts to the images of the four chamber view, with reported screening rates of $80 \%$ compared to $50 \%$ previously (10). After the CNEOF updated factors to be included in ultrasound screening in France in 2016, examination of the left ventricular outflow tract (LVOT) was added to ultrasound examination conducted in the second and third trimester of pregnancy.

The use of quality criteria for outflow tract scans addresses the need to minimize the source of misevaluation and/or misinterpretation of images of foetal heart anatomy, for this area of interest (8).

The objective of this study was, on the one hand, to carry out an audit of past and current practices and, on the other, to study possible modifications following the introduction of quality criteria for LVOT examination in the field of foetal echocardiography screening. It is crucial to rely on precise anatomical features which should be investigated and identified on such images.

\section{Materials and Methods}

We conducted a multicenter, retrospective and prospective, descriptive, longitudinal study between 2016 and 2020 .

\section{Data collection}

Our study focused on three periods:

- Before 2016 : to assess the use of LVOT images before the CNEOF update (introduction of the LVOT assessment).

- In 2017 : to assess practices after release of the update

- In the first quarter of 2020: to assess practices after the introduction of, and guidance regarding quality criteria for LVOT examination in the second half of 2019.

Our database consists of information from two tertiary centers (the Saint Joseph Hospital in Marseille and the Groupe Hospitalier Sud Réunion de Saint Pierre [GHSR]) and one primary center (an ultrasound center in the Var). Images were retrieved from records via the following:

- The Saint Joseph Hospital in Marseille: three doctors (one senior expert and two senior experienced obstetricians), and one midwife sonographer.

- The GHSR: three doctors (one senior expert, one senior experienced, and one junior obstetricians), and one midwife sonographer.

- The Var screening centre: one experienced senior radiologist and one midwife sonographer.

For each operator, we extracted, from the respective databases, images relating to the LVOT corresponding to 15 examinations in the second and third trimester, for all three periods. For each file that was randomly examined, there were either no images, or one or more images. For the latter, the best image for LVOT examination was selected.

The person responsible for retrieving these images referred to the operators as A (senior expert), B (senior experienced obstetrician), C (senior experienced obstetrician) and D (midwife) for the Saint Joseph hospital; 
A (senior expert), B (senior experienced obstetrician), C (young obstetrician) and D (midwife) for the GHSR; and A (senior experienced radiologist) and B (midwife) for the Var center. Three files (before 2016, in 2017, and in 2020) were thus created and contained data on the practitioners' examinations.

For each practitioner, there were 30 files, numbered T2-1 to T2-15 for the 15 examinations in the second trimester and T3-1 to T3-15 for the 15 examinations in the third trimester for each of the three periods.

\section{Data processing}

Data were based on seven LVOT quality criteria proposed by Dr. E. Quarello (EQ) in 2019 (11), defined by visualization of the ANNEX 1 .

From the very start of our data collection, we asked all practitioners to carefully read the information and explanatory sheets on the proposed quality criteria $(\boldsymbol{A N N \boldsymbol { N }} \mathbf{1})$. Three months later, we evaluated the possible implementation of these criteria in their daily practice through the study of images relating to examination of the LVOT from 15 examinations in the second trimester and 15 examinations in the third trimester, for these same sonographers during the first quarter of 2020.

Over the three periods, each image from the 10 practitioners' examinations was rated for each of the seven quality criteria. The ratings chosen were 0 or 1 depending on the absence or presence of the quality criteria, respectively. The rating was carried out by a resident specialising in gynaecology-obstetrics with a focus on foetal medicine, Maud Regouin (MR). Thus, each image was scored out of seven points.

For all the images from each period, we attempted to identify the position of the back of the foetus according to the following classification (Figure 1 ):

- Position 1: foetal back between 12 o'clock and 3 o'clock

- Position 2: foetal back between 3 o'clock and 6 o'clock

- Position 3: foetal back between 6 o'clock and 9 o'clock

- Position 4: foetal back between 9 o'clock and 12 o'clock

To analyze inter-operator variability, 30 images were then arbitrarily selected from the image bank and an expert (EQ) was asked to grade each of them. These scores were compared to the scores from the trainee doctor (MR) for the same set of images. To analyze intra-operative variability of the junior doctor and expert (EQ), the 30 images were analyzed again after more than 24 hours by the same respective doctors and the scores were compared for the same set of images.

\section{Data protection}

All images were collected anonymously in each database. Only data that was strictly necessary and relevant to the research objectives was collected. All images were anonymised by the practitioners by removing first and last names. Access to this database was confidential and restricted to the direct users of the data.

\section{Ethical and regulatory considerations}

As this was non-interventional retrospective and prospective research involving non-human data from a study in the health field, a simplified MR004 procedure was granted with an agreement to comply with the National Commission for Information Technology and Civil Liberties (CNIL) (CNIL Reference 2219789 v 0) and registration of the project on the Register of studies without CNIL authorization. Patients were informed individually and in writing that ultrasound data from their records could be used. Each patient was given the possibility of declining the use of their data, by any means possible. In the event of no reply, compliance was assumed.

Statistical analysis 
Image-quality scores were expressed as mean \pm standard deviation (SD) or mean (95\% confidence interval). Subgroups defined by trimester, time period, center, operator and cardiac position were compared using t-tests or ANOVA. When the ANOVA test was significant, a post-hoc test with Bonferroni correction was used for pairwise comparisons. When studied separately, quality criteria were expressed as a percentage for the three time periods and according to cardiac position.

To estimate intra-rater and inter-rater variability of the image-quality score, the intraclass correlation coefficient (ICC [95\% confidence interval]) was estimated based on single-rating, absolute-agreement, 2-way mixed(intra-rater) and random- (inter-rater) effects models. To estimate intra-rater and inter-rater variability of the individual (binary) quality criterion, Kappa coefficient was used.

All statistical analysis were carried out using IBM SPSS 20.0 for Windows software (IBM Inc., New York, USA). A value of $\mathrm{p}<0.05$ was considered statistically significant.

\section{Funding and conflicts of interest}

The authors confirm that they have no conflicts of interest in this study.

\section{Results}

For each practitioner, we analyzed 15 cases from the second trimester and 15 from the third trimester for the three periods, i.e ., 90 cases per practitioner, which represented 300 cases before 2016, 300 in 2017, and 300 in 2020 , making a total of 900 cases.

\section{Inclusion of LVOT images}

In our study, of the 900 files analyzed, there were no images relating to the LVOT in 51 (5.7\%) of the cases. These files were therefore given a score of 0 . These included $14(4.7 \%)$ before 2016, $17(5.7 \%)$ in 2017, and 20 $(6.7 \%)$ in $2020 ; 34 / 360(9.4 \%)$ were taken from files extracted from the database of the Saint Joseph hospital and 17/180 (9.4\%) from the Var center. For the Saint Joseph's Hospital, LVOT images were absent in 8/120 $(6.7 \%)$ before $2016,11 / 120(9.2 \%)$ in 2017 , and 15/120 (12.5\%) in 2020. For the Var center, LVOT images were absent in 6/60 (10\%) before 2016, $6 / 60(10 \%)$ in 2017, and 5/60 (8.3\%) in 2020. In total, 25/450 (5.6\%) images were documented to be absent in the second trimester and 26/450 (5.8\%) in the third trimester. Thus, images of LVOT were present in $95.3 \%, 94.3 \%$, and $93.3 \%$ of cases before 2016, in 2017, and after 2020, respectively, for the three centers combined.

In order to make our analysis of practices more meaningful, we deliberately chose to exclude all files without an LVOT image. We therefore restricted our study to files with an image of the LVOT in order to shed more light on the use, or otherwise, of the proposed quality criteria.

\section{Total average scores for the three centres over the three periods}

The average total scores for the quality criteria across all centers were 5.49/7 (95\% CI: 5.36-5.62), 5.91/7 (95\% CI: 5.80-6.03), and 5.70 (95\% CI: 5.58-5.82) for study periods before 2016, in 2017, and after 2020, respectively.

There was a significant difference between the scores obtained before 2016 and in $2017(p<0.001)$ and also between the scores before 2016 and in $2020(\mathrm{p}=0.039)$. There was no significant difference following the introduction of the quality criteria; 2017 vs. 2020, p $=0.054$ (Figure 2). 


\section{Average scores according to trimester}

Score averages were 5.75/7 (95\% CI: 5.65-5.85) and 5.65/7 (95\% CI: 5.55-5.75) in the second and third trimester, respectively(Figure 3) .

\section{Average scores according to cardiac position}

In Position 1 (with the back at between 12 o'clock and 3 o'clock), the average score was 5.59/7 (95\% CI: 5.45-5.73). In Position 2 (back between 3 o'clock and 6 o'clock), the average score was 5.99/7 (95\% CI:5.876.10). In Position 3 (back between 6 o'clock and 9 o'clock), the average score was 5.82/7 (95\% CI: 5.65-5.98). In Position 4 (back between 9 o'clock and 12 o'clock), the average score was 5.33/7 (95\% CI: 5.19-5.48) (Figure 4 ).

Comparing the score averages obtained according to the position of the back of the foetus, we found a statistically significant difference between Positions 1 and $2(p<0,01)$, between Positions 2 and $4(p<0,01)$, and between Positions 3 and $4(p<0,01)$.

\section{Average scores according to centre for the three periods combined}

The average score at the GHSR was 5.90/7 (95\% CI: 5.8-6), at the Saint Joseph's Hospital 5.41/7 (95\% CI: 5.29-5.53), and at the Var center 5.83/7 (95\% CI: 5.69-5.98) (Figure 5 ).

We note a significant difference between the scores from Saint Joseph's Hospital and the GHSR, but also between Saint Joseph's Hospital and the Var center $(p<0.01$ for each). There was no significant difference between average scores from the GHSR and the Var center $(p=1)$.

\section{Average scores according to centre for each of the three periods}

The average scores for the three centers over the three periods studied are summarized in Figure 6 .

\section{At the GHSR}

Before 2016, the average score was 5.67 (95\% CI: 5.49-5.85), in 2017 5.96/7 (95\% CI: 5.78-6.14) and in 2020 6.08/7 (95\% CI: 5.90-6.26).

\section{At Saint Joseph's Hospital}

Before 2016, the average score was 5.25/7 (95\% CI: 5.06-5.44), in $20175.76 / 7$ (95\% CI: 5.57-5.95) and in 2020 5.21/7 (95\% CI: 5.02-5.40).

\section{At the Var centre}

Before 2016, the average score was 5.57/7 (95\% CI: 5.30-5.84), in $20176.11 / 7$ (95\% CI: 5.84-6.38) and in 2020 5.82/7 (95\% CI: 5.55-6.09).

\section{Average scores according to operator for the three periods combined}

Average scores for the 10 practitioners in the three centers over the three periods combined are summarized in Figure 7 .

\section{At the GHSR}

The total average for Practitioner A was 5.63/7 (95\% CI: 5.43-5.84), and for Practitioner B was 6.03/7 (95\% CI: 5.85-6.22). The total average for Practitioner C was 5.79 (95\% CI: 5.58-5.99), and for Practitioner D was 6.16 (95\% CI: 5.95-6.36). 


\section{At Saint Joseph's Hospital}

The total average for Practitioner A was 5.45/7 (95\% CI: 5.23-5.68), and for Practitioner B was 5.24/7 (95\% CI: 4.98-5.51). The total average for Practitioner C was 5.37/7 (95\% CI: 5.10-5.64), and for Practitioner D was 5.54/7 (95\% CI: 5.31-5.77).

\section{At the Var centre}

The total average for Practitioner A was 5.59/7 (95\% CI: 5.35-5.84) and for Practitioner B was 6.03/7 (95\% CI: 5.87-6.20).

\section{Average scores according to practitioner for each of the three periods}

Average scores for the 10 practitioners over the three periods studied are summarized in Figure 8 .

\section{Individual study of the seven quality criteria (\%)}

The percentage of quality criteria considered in all three periods combined and according to the position of the back of the foetus is summarized in Table 1 .

Consideration of the seven quality criteria over the three periods studied and according to the position of the back of the foetus is summarized in Figure 9 and Figure 10, respectively.

\section{Variability}

The intra-class correlation coefficient (ICC) for intra- and inter-operator agreement between the junior doctor (MR) and expert doctor (EQ), with and without 0 scores, is presented in Table 2 . All ICCs were between 0.601 and 1.

\section{Discussion}

The operators appear to have systematically included LVOT images in their screening practices, as images of the LVOT were found in $95 \%$ of the files studied. With regards to image quality, practices have improved since 2017, but there was no difference over the study period (first quarter of 2020) since the introduction of the quality criteria. Practitioners who were already aware of the use of LVOT images before the new recommendations continued to use them systematically, and probably more accurately after 2016. Scores were not significantly higher in the second trimester relative to the third trimester. Given the results of the Var center, echocardiography screening activity in the primary center is just as satisfactory as in the tertiary center. The scores obtained according to cardiac position show a significant improvement in average scores for Positions 2 and 3 when the back of the foetus is in the posterior position between 3 and 9 o'clock. It was noted that the Quality Criteria 5, 6 and 7 were very poorly considered over the three periods, regardless of cardiac position.

In addition to obtaining the required images, it is relevant to carry out a "quality" screening examination. This notion of quality presupposes that the images obtained are as informative as possible. As early as 2003, Chaoui advocated quality control of foetal echocardiograms in order to avoid inadequate examinations (7). Certification, similar to that required for first-trimester assessments, could be considered in an attempt to improve screening. Indeed, in some countries, regular audits for maintaining certification or quality control have been successful (12)(13)(14). Since then, the notion of quality control and auditing has developed in the field of echocardiography, with first-trimester cardiac screening examinations in low-risk populations (15), 
as well as cardio-thoracic ratio measurement (16), the use of four-chamber views (17)(18) and the outflow tracts $(17)(19)$. With regards to the 4-chamber view, apical or basal views are recommended in order to respect the quality criteria proposed(18). However, to a lesser extent, side views are also recommended. For the LVOT, one of the most important criteria is visualization of the interventricular septum and, in particular, its relationship to the possible overlying vessel. This area of interest is best visualized in 2D, when the ultrasound beam approaches it perpendicularly and not, as is too often the case, by taking a perpendicular approach to the lower part of the interventricular septum (Figure 11 ), with the risk of misinterpreting interventricular communication of the conical region, as shown by our example in Figures 12 and 13 . This principle of taking a perpendicular approach to the anatomical region on either side of the aortic valves is fundamental, whether the approach is anterior or posterior. It is therefore preferable to use a lateral approach to the LVOT. This underlines the importance of a dynamic examination by means of scanning and angulation methods for probes producing the ultrasound beam (5)(11)(20) and this point was also already highlighted by Sklansky and De Vore (8) .

The detection of conotruncal anomalies should and could be improved (21). This leads us to question the causes of such errors during ultrasound screening. Several factors, unrelated to the operator, have already been highlighted by different teams: gestational age at the time of ultrasound, maternal adipose tissue, history of abdominal surgery, foetal position, quantity of amniotic fluid, position of the placenta, etc. (22)(23). Other limiting factors directly attributable to sonographers have also been demonstrated: the experience of the operator (23), the number of ultrasound scans carried out annually, the inability to obtain the required images or so-called "quality" images and the inability of the operator to recognize unusual images despite obtaining quality anatomical images. The latter concepts have again been reported recently by Sun et al. (24) and Van Nisselrooij et al.(9), who also confirm that the main current limitation of CHD screening is linked to in poor image quality for other outflow tracts.

In the light of these observations and the relative consistency in reported CHD screening, it would appear to be appropriate to accept the extent of training programmes for sonographers, however, it is essential to propose solutions which should accompany these programmes. It is with this in mind that Artificial Intelligence (AI) has been developed. Currently, the most relevant model in automated learning of image analysis is the convolutional neural network (25)(26). AI could thus help the practitioner by carrying out a real-time audit of the images provided (presence or absence and quality), helping to obtain recommended quality anatomical images (27), but also in terms of improving image resolution (28)(29)(30). AI could thus be of great help, not from a diagnostic perspective, but rather as a support tool for the practitioner and a guarantee of quality, analogous to a seatbelt in a car, as it may be associated with a potentially high negative predictive value regarding the risk of major CHD.

\section{The strengths of our study}

. This was a multicenter study based in different centers with practitioners from different backgrounds and with different levels of experience, providing an example of current screening practices in the same country in tertiary and primary centers.

. This work focused on the quality of echocardiographic screening and the causes associated with the current limitations of screening for CHD.

. Inter- and intra-operator variability regarding the proposed criteria was low, as the kappa coefficients were all reported at between 0.601 and 1. Concordance ranged from good to very good, thus the scoring of the images was carried out correctly and the proposed criteria were easy to use.

. The evaluation of the quality criteria proposed was carried out in routine practice by operators who probably have different degrees of motivation in their desire to use new tools aimed at improving screening practices. Indeed, most of the studies relating to the study of quality criteria were carried out in tertiary centers with operators working in close contact with the person(s) capable of ascertaining the quality criteria. We thus 
acknowledge that the reality may be quite different, and this sheds new light on what underlies the current limitations of CHD screening.

. Quality criteria for LVOT screening should be distinguished from normality criteria. For example, for Criterion 6, the quality criterion is not the presence of septo-aortic continuity but the visualization of the relationship between the interventricular septum and the possible overlying vessel, whether or not there is continuity between the interventricular septum and this possible vessel.

\section{Limitations of the study}

. It is not possible to know whether the lack of images in the files reflects a lack of investigation of the area in question, or whether the area was investigated but no image was available and stored to demonstrate this.

. Does a significant difference in quality score truly reflect a lack of, or a real change in practice, with a real clinical impact? Indeed, assessment of the only relevant difference would be based on rates of detection of heart defects recorded in registers.

. The lack of significant improvement in scores after the introduction of the quality criteria raises questions about their relevance and use, but also about the techniques associated with guidance on how to implement them. Practitioners may have been made aware of the contribution of the quality criteria even if this was not reflected in the analysis. It is clear that we should try to understand the causes behind the non-compliance of these criteria: difficulty in understanding them, in remembering to use them and/or being aware of them.

\section{Conclusion}

Over the three periods in our study, the left ventricular outflow tract is mainly carried out. The quality scores remained globally stable since the updating of the CNEOF recommendations in 2016, despite the introduction in 2019 of quality criteria in an attempt to improve practices related to obtaining LVOT images. No significant change in practices was noted in the first quarter of 2020 , following the introduction of the LVOT quality criteria. Finally, the main limiting cause of the lack of improvement in cardiac screening is probably human, due to possible lack of interest on the part of operators, and/or a lack of appropriation for the quality criteria, and/or errors in the choice of quality criteria proposed (too complex).

Analysis of the foetal heart remains challenging, mainly due to its complex anatomy, the small size of the areas studied, heart movements, involuntary foetal movements, and the lack of expertise in foetal echocardiography among some sonographers. Therefore, the use of new Artificial Intelligence-supported technologies aimed at making screening echocardiography practices safer seems likely to be the next step towards precision medicine in the future $(25)(31)$.

Conflicts of interest: the authors report no conflict of interest.

\section{Credit Author Statement:}

Maud REGOUIN: Writing, Legal processing, Formal analysis

Julien MANCINI: Statistics analysis

Antoine LAFOUGE: Data collection

Coralie DUMONT: Data collection

Nathalie FONTAINE: Data collection

Stéphanie ROUSSIN: Data collection

Jimmy GUICHARD: Data collection 
Edwin QUARELLO: Data collection, Formal analysis, Conceptualization, Methodology, Supervision, Writing, Reviewing, and Editing

Ethics Approval: As this was non-interventional retrospective and prospective research involving nonhuman data from a study in the health field, a simplified MR004 procedure was granted with an agreement to comply with the National Commission for Information Technology and Civil Liberties (CNIL) (CNIL Reference $2219789 \mathrm{v} 0$ ) and registration of the project on the Register of studies without CNIL authorization. Patients were informed individually and in writing that ultrasound data from their records could be used. Each patient was given the possibility of declining the use of their data, by any means possible. In the event of no reply, compliance was assumed.

Source of funding: None

Abstract words count: 250

Main text words count: 3701

Legends

Annex 1: Quality criteria of the Left Ventricular Outflow Tract assessment

1. A Left ventricular cavity (whether anatomically on the left or right) and identification to the tip of the ventricle and interventricular septum.

2. The presence of a valvular ring and the origin of the overlying vessel, whether or not this ventricle gives rise to a vessel.

3. An anechogenic Space Above And In The Middle Of The Semilunar Valves (Visualisation And position above the semilunar valves), corresponding to the anterosuperior part of the ventricular cavity on the right.

4. An anechogenic space located below the semilunar valves (visualisation and position below the semilunar valves), corresponding to the atrium located on the left and behind the left atrioventricular valve.

5. The relationship between the left atrioventricular valve and the overlying vessel: continuity or discontinuity between the atrioventricular valve and the overlying vessel.

6. The relationship between the interventricular septum and the overlying vessel: continuity or discontinuity between the septum and the overlying vessel.

7. Visualisation of the semilunar valves: normal or dysplastic in appearance.

8: Thoracic aorta belongs not to the quality criteria

Figure 1: Schematic representation of the LVOT according to the position of the fetal back following to the classification

- Position 1: foetal back between 12 o'clock and 3 o'clock

- Position 2: foetal back between 3 o'clock and 6 o'clock

- Position 3: foetal back between 6 o'clock and 9 o'clock

- Position 4: foetal back between 9 o'clock and 12 o'clock

\section{Figure 2: Total average scores for the three centres over the three periods}

The average total scores for the quality criteria across all centres were 5.49/7 (95\% CI: 5.36-5.62), 5.91/7 (95\% CI: 5.80-6.03), and 5.70 (95\% CI: 5.58-5.82) for study periods before 2016, in 2017, and after 2020, respectively.

There was a significant difference between the scores obtained before 2016 and in $2017(p<0.001)$ and also between the scores before 2016 and in $2020(\mathrm{p}=0.039)$. There was no significant difference following the introduction of the quality criteria; 2017 vs. $2020, \mathrm{p}=0.054$ 


\section{Figure 3: Average scores according to trimester}

Score averages were 5.75/7 (95\% CI: 5.65-5.85) and 5.65/7 (95\% CI: 5.55-5.75) in the second and third trimester respectively.

\section{Figure 4: Average scores according to cardiac position}

In Position 1 (with the back at between 12 o'clock and 3 o'clock), the average score was 5.59/7 (95\% CI: 5.45-5.73). In Position 2 (back between 3 o'clock and 6 o'clock), the average score was 5.99/7 (95\% CI:5.876.10). In Position 3 (back between 6 o'clock and 9 o'clock), the average score was 5.82/7 (95\% CI: 5.65-5.98). In Position 4 (back between 9 o'clock and 12 o'clock), the average score was 5.33/7 (95\% CI: 5.19-5.48).

Comparing the score averages obtained according to the position of the back of the foetus, we found a statistically significant difference between Positions 1 and $2(p<0,01)$, between Positions 2 and $4(p<0,01)$, and between Positions 3 and $4(p<0,01)$.

\section{Figure 5: Average scores according to centre for the three periods combined}

The average score at the GHSR was 5.90/7 (95\% CI: 5.8-6), at the Saint Joseph's Hospital 5.41/7 (95\% CI: 5.29-5.53), and at the Var centre 5.83/7 (95\% CI: 5.69-5.98)

\section{Figure 6: Average scores according to centre for each of the three periods}

Figure 7: Average scores according to operator for the three periods combined

Figure 8: Average scores according to practitioner for each of the three periods

Table 1: The percentage of quality criteria considered in all three periods combined and according to the position of the back of the foetus

Figure 9: The percentage of quality criteria considered in all three periods.

Figure 10: The percentage of quality criteria considered according to the position of the back of the foetus.

Table 2: The intra-class correlation coefficient (ICC) for intra- and inter-operator agreement between the junior doctor (MR) and expert doctor (EQ), with and without 0 scores

Figure 11: Representation of the ultrasonographic beam (arrows) perpendicular to the inferior (a1 and a2) and the superior (b1 and b2) part of the interventricular septum represented respectively by a continuous line and dotted line.

Figure 12: Ultrasonographic views at 16 (a) and 22 (b) weeks of the LVOT. The superior part of the interventricular septum was never approached in a perpendicular way. It was possible to suspect a communication at 16 weeks but at 22 weeks this area was not zoomed appropriately and remained blurred.

Figure 13: Postnatal ultrasonographic assessment of the superior part of the interventricular septum of the foetus displayed in Figure 12. A large VSD was present and missed prenatally.

\section{References}

1. Bensemlali M, Bajolle F, Laux D, Parisot P, Ladouceur M, Fermont L, et al. Neonatal management and outcomes of prenatally diagnosed CHDs. Cardiol Young. mars 2017;27(2):344-53.

2. Bonnet D, Coltri A, Butera G, Fermont L, Le Bidois J, Kachaner J, et al. Detection of transposition of the great arteries in fetuses reduces neonatal morbidity and mortality. Circulation. 23 fevr 1999;99(7):916-8. 
3. Calderon J, Angeard N, Moutier S, Plumet M-H, Jambaque I, Bonnet D. Impact of prenatal diagnosis on neurocognitive outcomes in children with transposition of the great arteries. J Pediatr. juill 2012;161(1):9498.e1.

4. Kasmi L, Bonnet D, Montreuil M, Kalfa D, Geronikola N, Bellinger DC, et al. Neuropsychological and Psychiatric Outcomes in Dextro-Transposition of the Great Arteries across the Lifespan: A State-of-the-Art Review. Front Pediatr. 2017;5:59.

5. International Society of Ultrasound in Obstetrics and Gynecology null, Carvalho JS, Allan LD, Chaoui R, Copel JA, DeVore GR, et al. ISUOG Practice Guidelines (updated): sonographic screening examination of the fetal heart. Ultrasound Obstet Gynecol. mars 2013;41(3):348-59.

6. Sharland G. Routine fetal cardiac screening: what are we doing and what should we do? Prenat Diagn. 30 dec 2004;24(13):1123-9.

7. Chaoui R. The four-chamber view: four reasons why it seems to fail in screening for cardiac abnormalities and suggestions to improve detection rate. Ultrasound Obstet Gynecol. juill 2003;22(1):3-10.

8. Sklansky M, DeVore GR. Fetal Cardiac Screening: What Are We (and Our Guidelines) Doing Wrong? J Ultrasound Med. avr 2016;35(4):679-81.

9. Nisselrooij AEL, Teunissen AKK, Clur SA, Rozendaal L, Pajkrt E, Linskens IH, et al. Why are congenital heart defects being missed? Ultrasound Obstet Gynecol. juin 2020;55(6):747-57.

10. Carvalho JS, Mavrides E, Shinebourne EA, Campbell S, Thilaganathan B. Improving the effectiveness of routine prenatal screening for major congenital heart defects. Heart. oct 2002;88(4):387-91.

11. Quarello E. [The left outflow tract: Normal features]. Gynecol Obstet Fertil Senol. sept 2019;47(9):680-8.

12. Pinto NM, Henry KA, Grobman WA, Ness A, Miller S, Ellestad S, et al. Physician Barriers and Facilitators for Screening for Congenital Heart Disease With Routine Obstetric Ultrasound: A National United States Survey. J Ultrasound Med. juin 2020;39(6):1143-53.

13. Pinto NM, Henry KA, Wei G, Sheng X, Green T, Puchalski MD, et al. Barriers to Sonographer Screening for Fetal Heart Defects: A U.S. National Survey. Fetal Diagn Ther. 2020;47(3):188-97.

14. Pinto NM, Morris SA, Moon-Grady AJ, Donofrio MT. Prenatal cardiac care: Goals, priorities \& gaps in knowledge in fetal cardiovascular disease: Perspectives of the Fetal Heart Society. Progress in Pediatric Cardiology. oct 2020;101312.

15. Quarello E, Lafouge A, Fries N, Salomon LJ, CFEF. Basic heart examination: feasibility study of firsttrimester systematic simplified fetal echocardiography. Ultrasound Obstet Gynecol. fevr 2017;49(2):224-30.

16. Awadh AMA, Prefumo F, Bland JM, Carvalho JS. Assessment of the intraobserver variability in the measurement of fetal cardiothoracic ratio using ellipse and diameter methods. Ultrasound Obstet Gynecol. juill 2006;28(1):53-6.

17. Sairam S, Awadh AMA, Cook K, Papageorghiou AT, Carvalho JS. Impact of audit of routine second-trimester cardiac images using a novel image-scoring method. Ultrasound Obstet Gynecol. mai 2009;33(5):545-51.

18. Jaudi S, Fries N, Tezenas du Montcel S, Dommergues M. The impact of insonation angle on four-chamber view image quality: an observational study on 2866 routine scans: Fetal heart insonation. Prenat Diagn. avr 2015;35(4):382-5.

19. Salomon LJ, Winer N, Bernard JP, Ville Y. A score-based method for quality control of fetal images at routine second-trimester ultrasound examination. Prenat Diagn. sept 2008;28(9):822-7.

20. Yagel S, Cohen SM, Achiron R. Examination of the fetal heart by five short-axis views: a proposed screening method for comprehensive cardiac evaluation. Ultrasound Obstet Gynecol. mai 2001;17(5):367-9. 
21. van Velzen CL, Ket JCF, van de Ven PM, Blom NA, Haak MC. Systematic review and meta-analysis of the performance of second-trimester screening for prenatal detection of congenital heart defects. Int $\mathrm{J}$ Gynaecol Obstet. fevr 2018;140(2):137-45.

22. Bartoli A, Quarello E, Voznyuk I, Gorincour G. [Artificial intelligence and fetal imaging: What are we talking about?]. Gynecol Obstet Fertil Senol. nov 2019;47(11):765-8.

23. Tegnander E, Eik-Nes SH. The examiner's ultrasound experience has a significant impact on the detection rate of congenital heart defects at the second-trimester fetal examination. Ultrasound Obstet Gynecol. juill $2006 ; 28(1): 8-14$.

24. Sun HY, Proudfoot JA, McCandless RT. Prenatal detection of critical cardiac outflow tract anomalies remains suboptimal despite revised obstetrical imaging guidelines. Congenital Heart Disease. sept 2018;13(5):748-56.

25. Garcia-Canadilla P, Sanchez-Martinez S, Crispi F, Bijnens B. Machine Learning in Fetal Cardiology: What to Expect. Fetal Diagn Ther. 2020;47(5):363-72.

26. Arnaout R, Curran L, Chinn E, Zhao Y, Moon-Grady A. Deep-learning models improve on communitylevel diagnosis for common congenital heart disease lesions. arXiv:180906993 [cs] [Internet]. 18 sept 2018 [cite 17 janv 2021]; Disponible sur: http://arxiv.org/abs/1809.06993

27. Muse ED, Topol EJ. Guiding ultrasound image capture with artificial intelligence. Lancet. 12 sept 2020;396(10253):749.

28. Harvey H, Topol EJ. More than meets the AI: refining image acquisition and resolution. The Lancet. nov 2020;396(10261):1479.

29. Milstein A, Topol EJ. Computer vision's potential to improve health care. The Lancet. mai 2020;395(10236):1537.

30. Mozumdar N, Rowland J, Pan S, Rajagopal H, Geiger MK, Srivastava S, et al. Diagnostic Accuracy of Fetal Echocardiography in Congenital Heart Disease. Journal of the American Society of Echocardiography. nov 2020;33(11):1384-90.

31. Dey D, Slomka PJ, Leeson P, Comaniciu D, Shrestha S, Sengupta PP, et al. Artificial Intelligence in Cardiovascular Imaging: JACC State-of-the-Art Review. J Am Coll Cardiol. 26 mars 2019;73(11):1317-35.
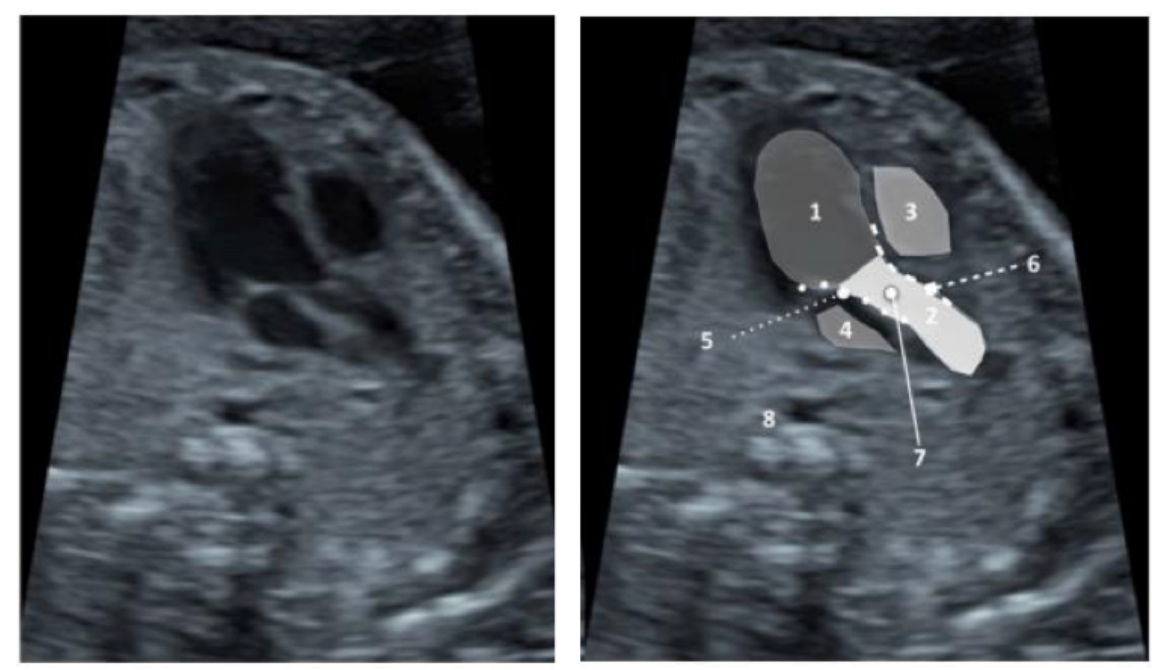

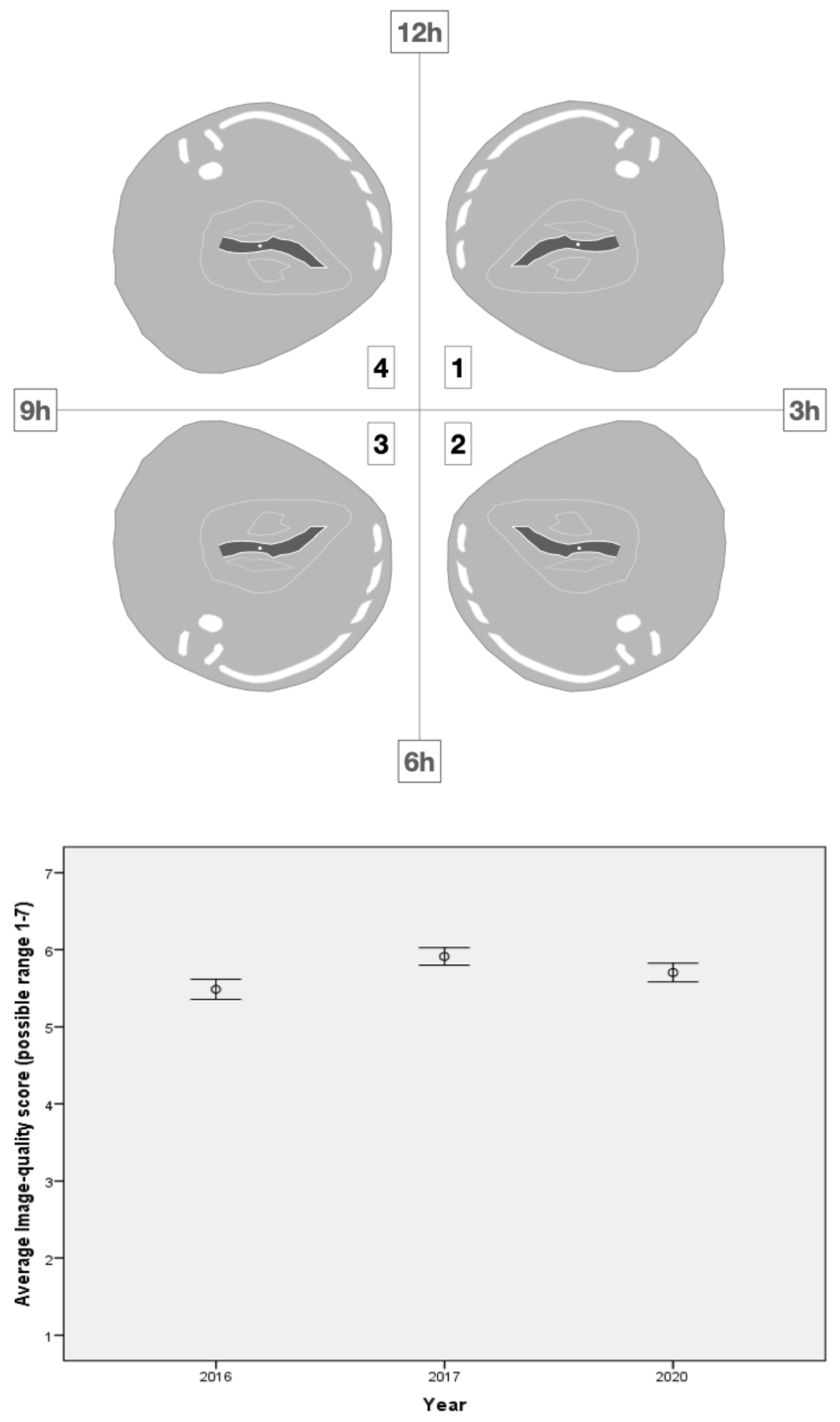

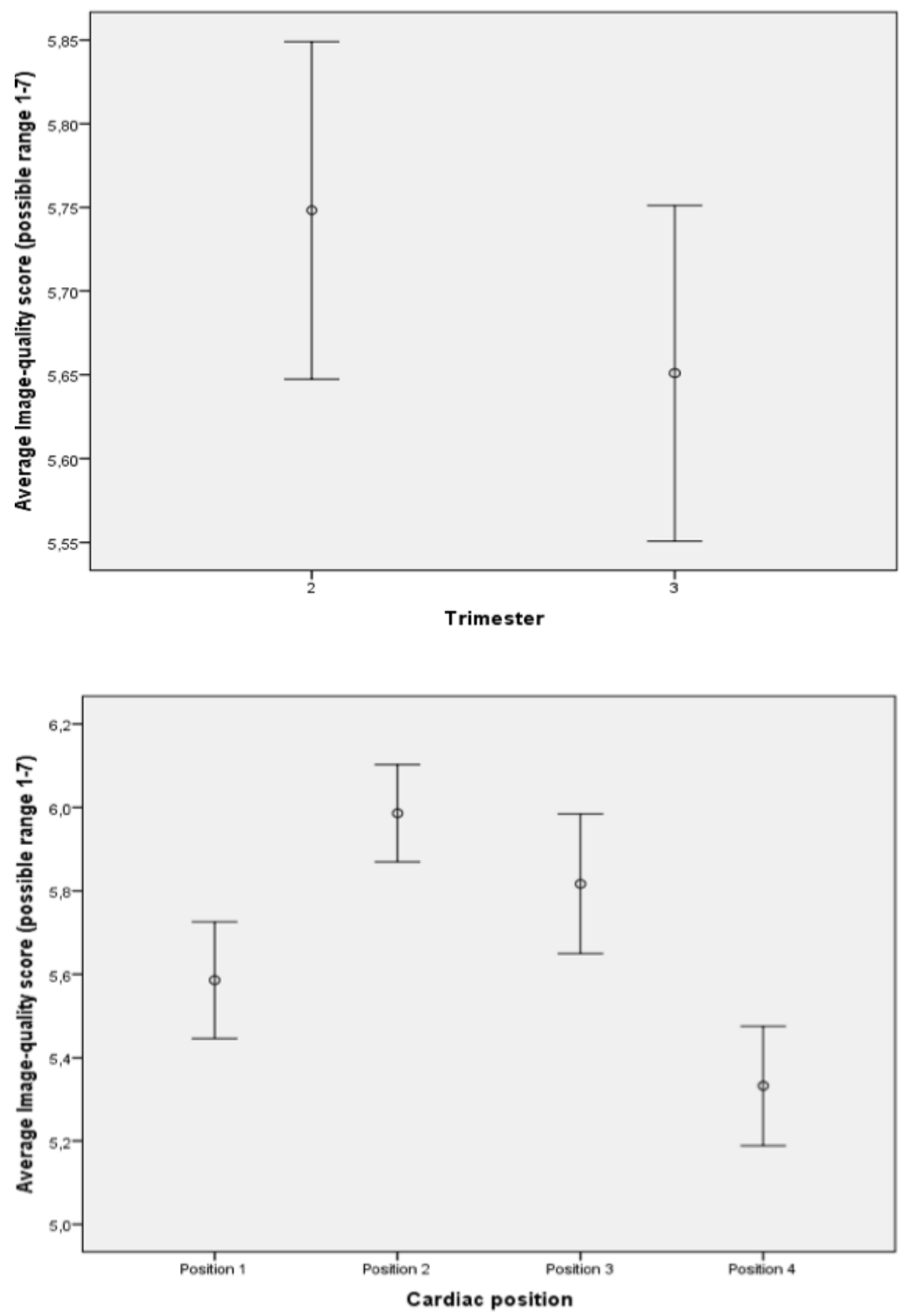

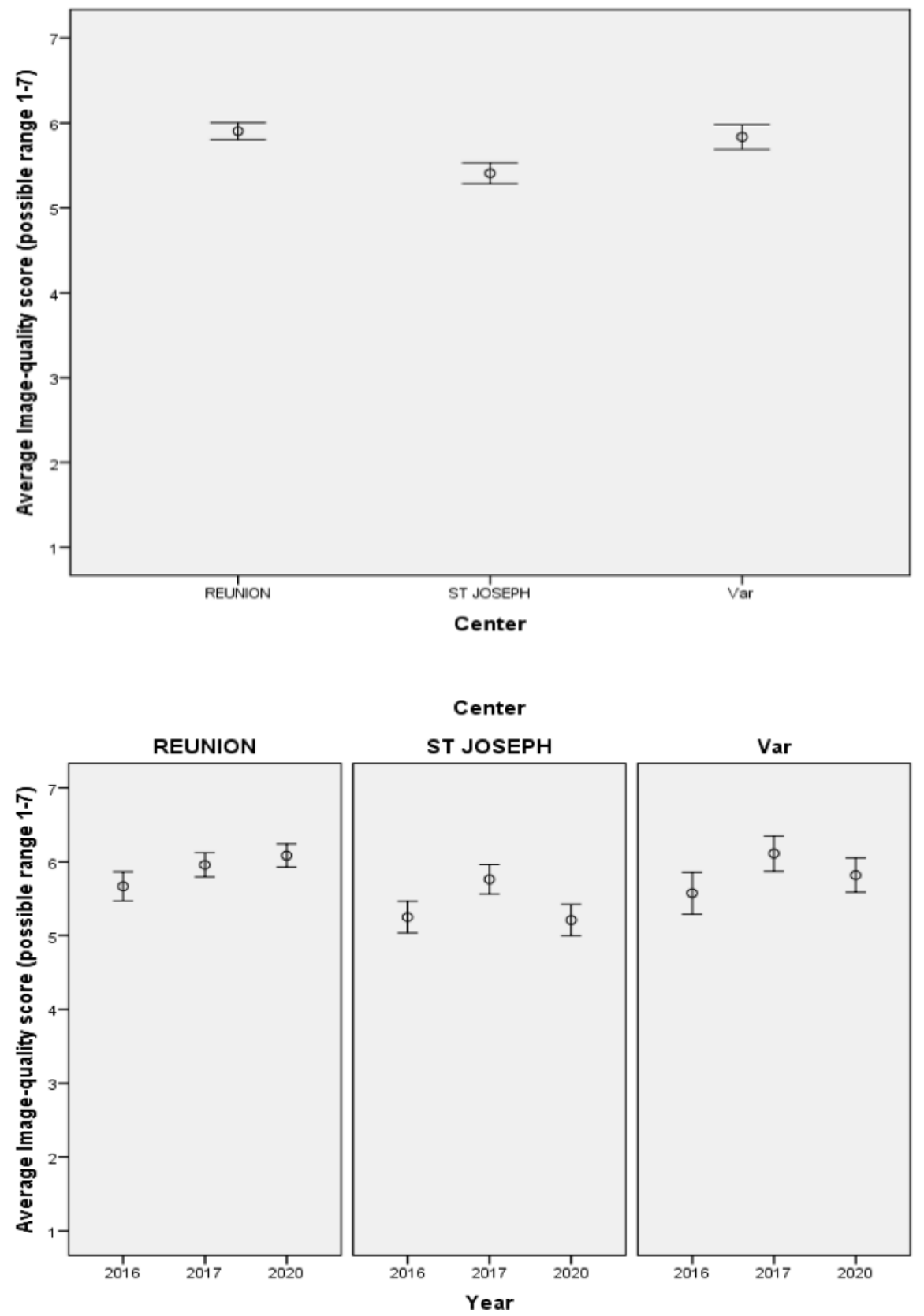

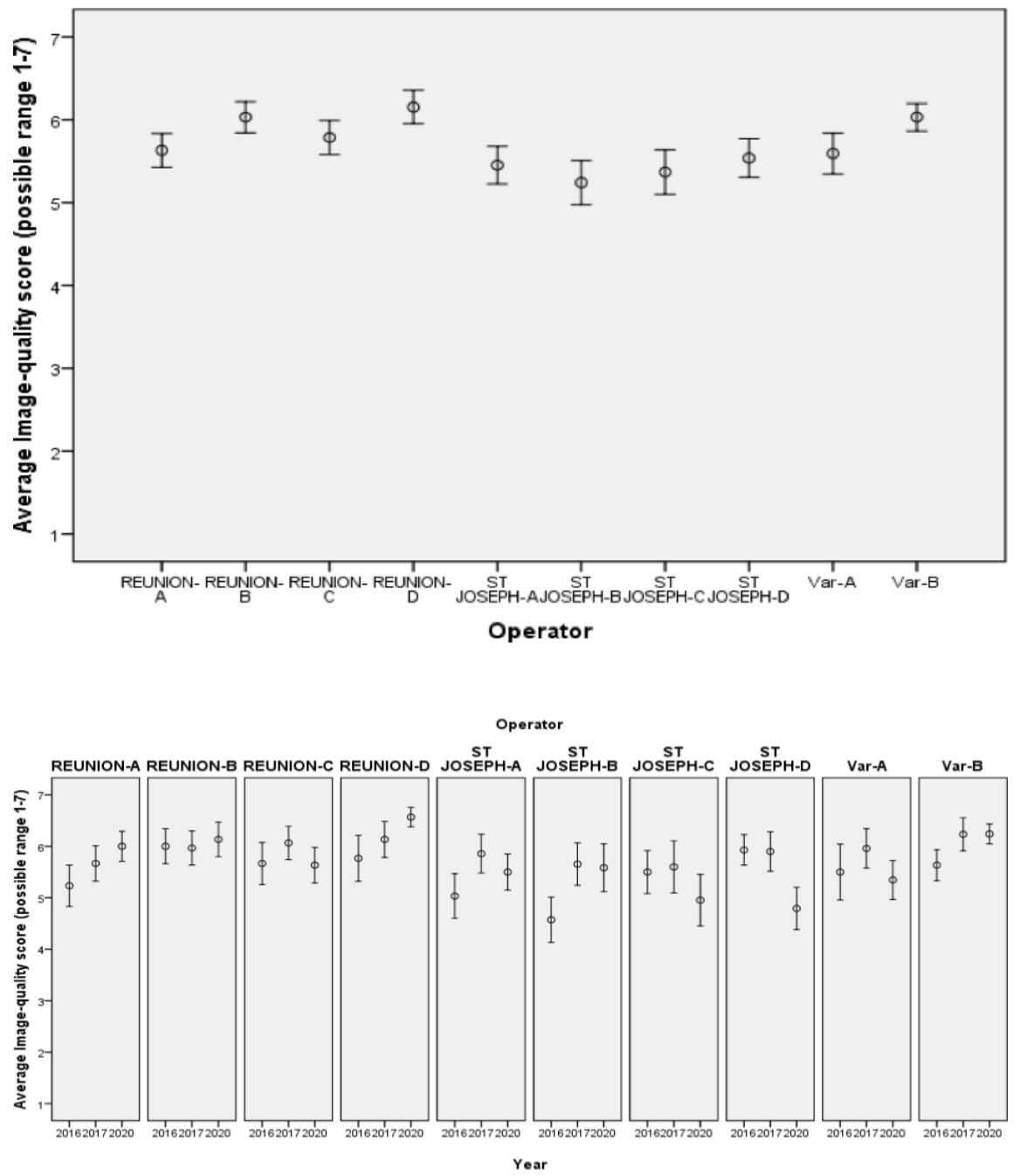

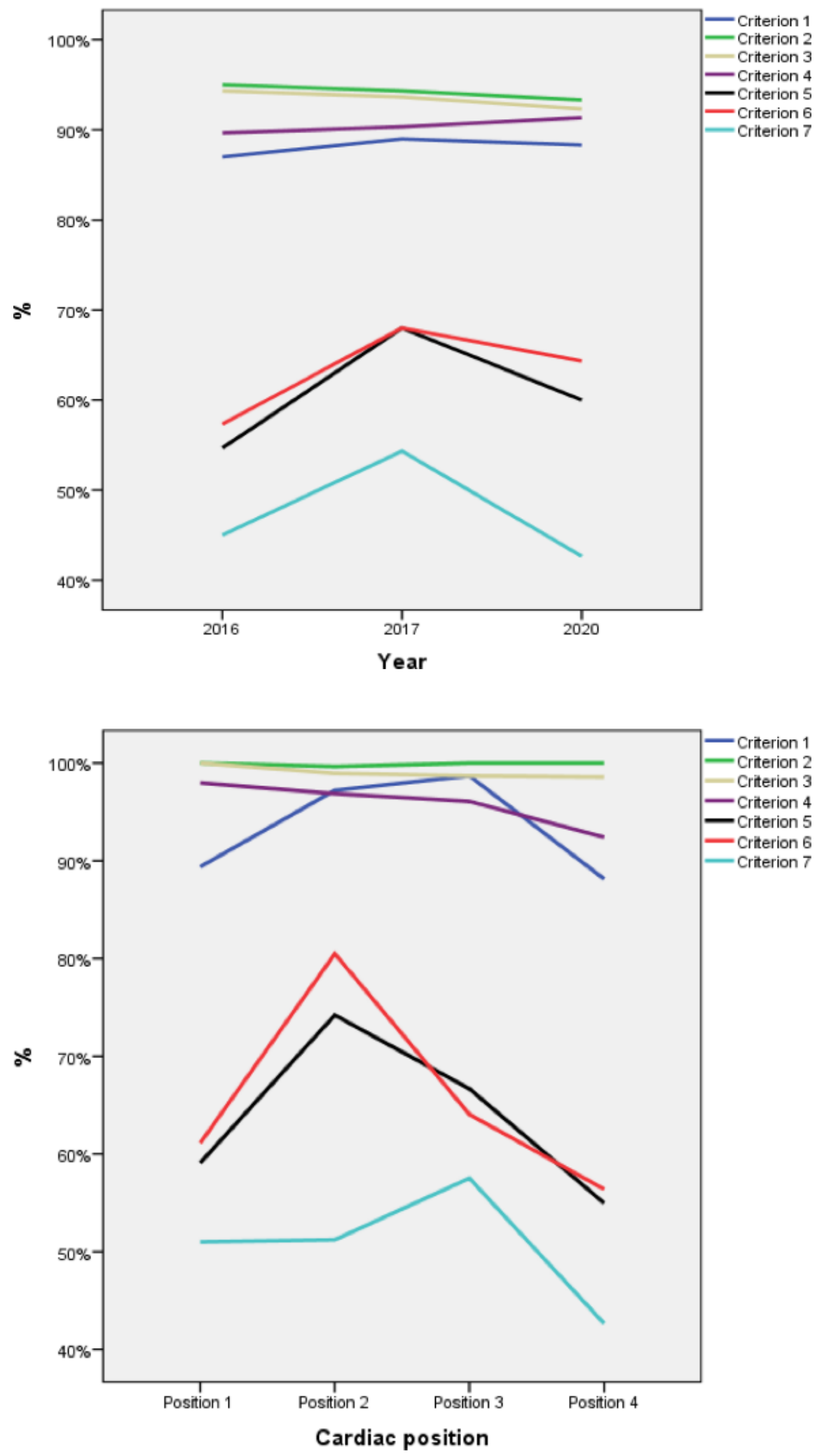

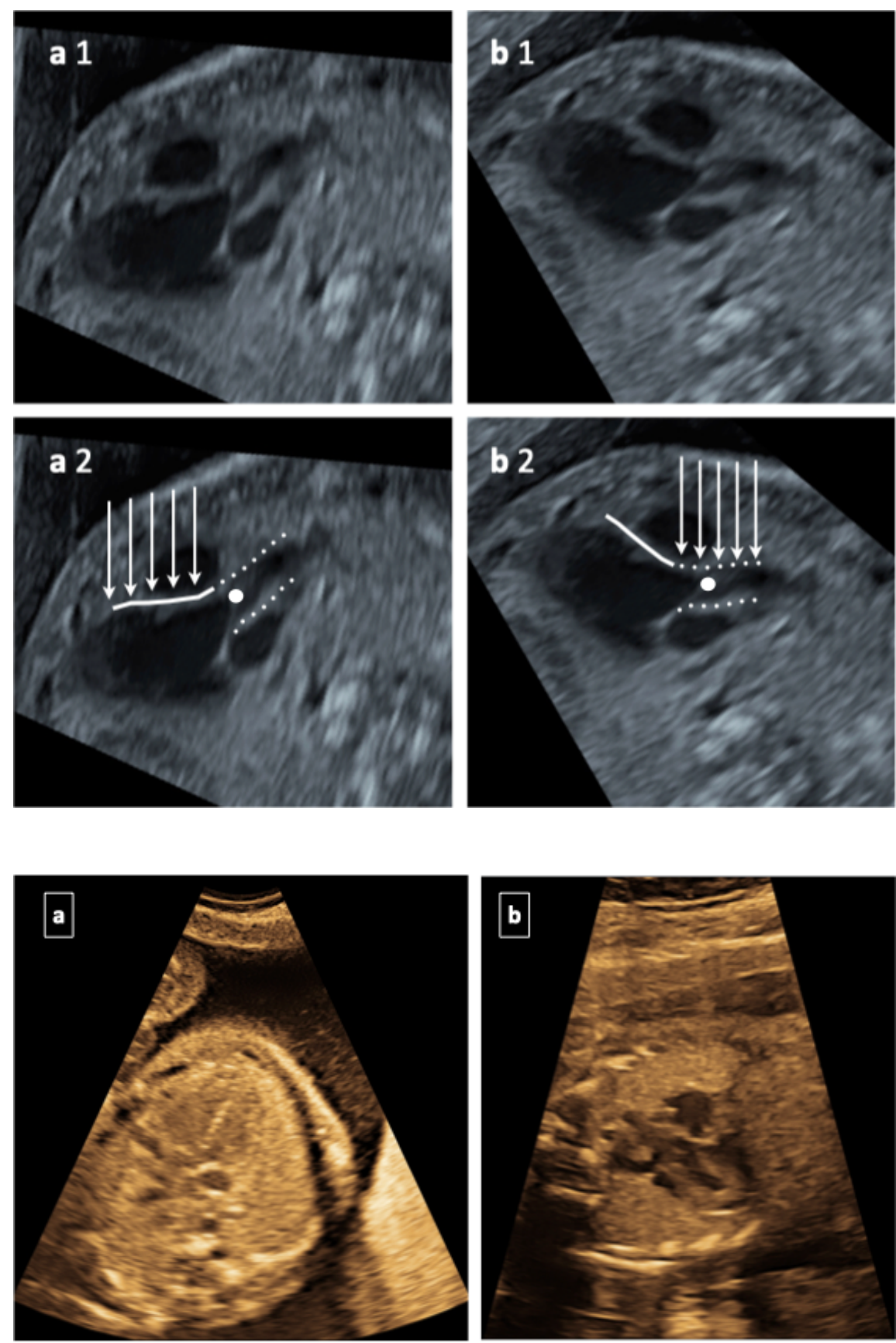


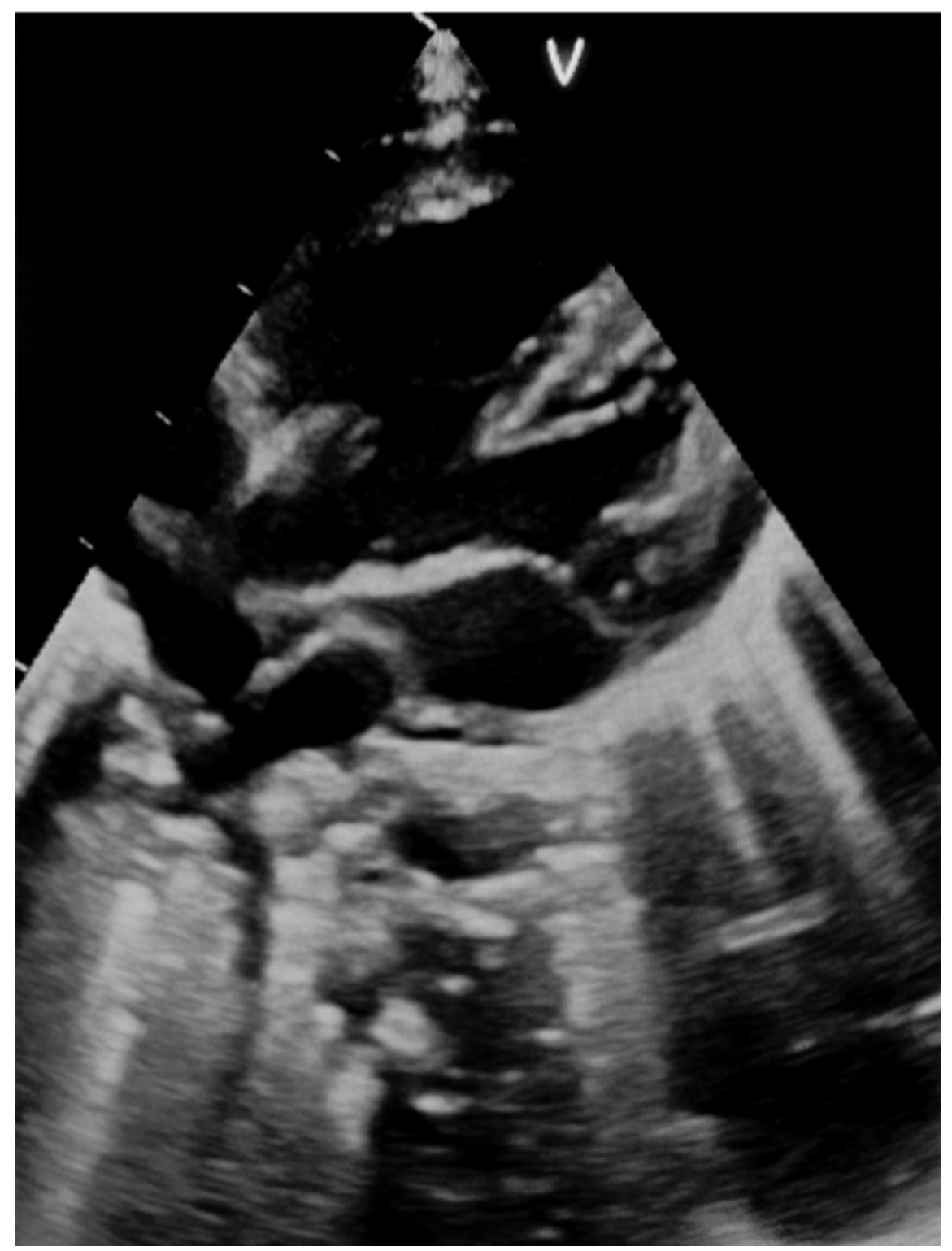

\section{Hosted file}

Table 1.pdf available at https://authorea.com/users/401073/articles/513313-the-left-outflowtract-in-foetal-echocardiography-screening-an-audit-of-practices-and-quality-criteria

\section{Hosted file}

Table 2.pdf available at https://authorea.com/users/401073/articles/513313-the-left-outflowtract-in-foetal-echocardiography-screening-an-audit-of-practices-and-quality-criteria 